\title{
Article
}

\section{To formulate or not to formulate? A case study}

Houghton, Karrie and Jones, Emma

Available at http://clok.uclan.ac.uk/17468/

Houghton, Karrie and Jones, Emma ORCID: 0000-0002-2153-2781 (2016) To formulate or not to formulate? A case study. British Journal of Mental Health Nursing, 5 (6). pp. 274-281. ISSN 2049-5919

It is advisable to refer to the publisher's version if you intend to cite from the work. 10.12968/bjmh.2016.5.6.274

For more information about UCLan's research in this area go to http://www.uclan.ac.uk/researchgroups/ and search for < name of research Group>.

For information about Research generally at UCLan please go to http://www.uclan.ac.uk/research/

All outputs in CLoK are protected by Intellectual Property Rights law, including Copyright law. Copyright, IPR and Moral Rights for the works on this site are retained by the individual authors and/or other copyright owners. Terms and conditions for use of this material are defined in the policies page.

\section{CLoK}

Central Lancashire online Knowledge www.clok.uclan.ac.uk

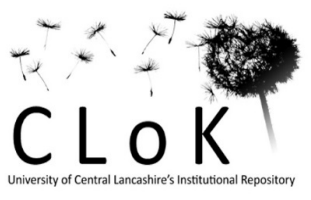




\section{Full title:}

Exploration of the assessment, formulation and interventions used in a secure service working with service users with a personality disorder: A case study.

\section{Short title:}

To formulate or not to formulate?

\section{Abstract:}

Abstract: Personality disorders are common within specialist forensic mental health services and can complicate the treatment and care of people who may also have other co-morbid mental health problems; especially because they are based on unclear and unvalidated descriptions. The following article analyses the case of Laura who is a fictional character that represents the presentation of several women who have received care for personality disorder(s) and other mental health problems in a specialist forensic hospital. The assessment, formulation and care planning process conducted with Laura adhered to evidence based practice guidelines and led to reductions in aggressive behaviour and violent incidents. This case study shows how effective case formulation can be used to understand service users with complex problems including personality disorder(s) and enhance the safe and effective care provided. Overall this case highlights the necessity of consistent staff teams and clinical supervision when working with this client group.

\section{$\underline{\text { Key phrases }}$}

1. This article will showcase the usefulness of case formulation when working with personality disorder

2. This case study will outline the care provided for women with severe personality disorders

3. This article will show how vital clinical supervision is for staff members working with personality disorders

\section{Classification of the paper:}

Case study 


\section{Introduction}

Personality disorder is defined as entrenched difficulties in inter-personal functioning, cognition and impulse control. People with personality disorders have often developed enduring maladaptive personal attributes that persistently affect every aspect of their life. (American Psychiatric Association (APA), 2013; WHO, 1992). It has been estimated that the weighted prevalence of personality disorder in the adult population in the UK is approximately 4.4\% (Coid et al., 2006). Personality disorders are controversial and there are many critiques surrounding the evidence base supporting their existence and thus the validity of the diagnosis and its criteria (Skodol et al., 2013).

Despite these issues in diagnosis, many people are given the label of personality disorder and are treated in mental health services. This case will consider the benefits of using formulation as a tool to guide the choice of interventions for people with personality disorders, by focusing on Laura who is a fictional character that represents the presentation of several women who received care on a personality disorder unit. In addition, the way in which mental health professionals worked with Laura will be reviewed including whether her personality disorder label affected this care. Finally, the assessment and interventions Laura received will be analysed and compared to evidence based treatment guidelines to understand whether treatment guidelines were adhered to and offer explanations/recommendations if they were not. All service user, ward and trust details will be anonymised throughout in order to preserve confidentiality (Nursing and Midwifery Council (NMC), 2015).

\section{Service Context}

Laura was transferred to a medium secure forensic unit- via section 48/49 of the Mental Health Act (MHA) (1983) - from prison where she had been remanded in custody pending a court appearance. There were seven women on the unit including Laura, all of whom had diagnoses of personality disorders as well as other mental health problems such as schizophrenia.

The staff on the unit often expressed that they felt as though there needed to be higher staffing numbers, particularly to support times when there was a higher acuity on the unit. Furthermore, difficulties in recruiting and retaining qualified nursing staff has been highlighted as a national issue within health care services (Gilburt, 2015); this could also be seen within this unit where there was often only one qualified nurse per shift. This is contrary to the Royal College of Psychiatrists (2010) advice that each mental health ward should have more than one qualified nurse per shift to provide safe and effective care. However, there was a well-established multi-disciplinary team (MDT) and a small number of regular nurses and health care assistants who had knowledge and experience of the unit. These staffing problems presented a significant challenge for conducting the assessment and treatment process with Laura. This unit was based within a wider hospital that contained 13 separate units, an occupational therapy centre, horticultural facilities and a social centre. There was also provision for psychological therapies and group sessions. However within the hospital some of the waiting lists for these sessions and facilities were lengthy and the lack of staffing affected whether the women could access these resources, as is a common occurrence 
in these types of settings (Lean et al., 2015).

\section{The Case}

Formulation was the main tool used in understanding Laura's situation because it values the individual's history, influences and situation in order to provide meaningful, recoveryfocused care (Aveline, 1999; British Psychological Society, 2011; MacNeil et al., 2012). However, it is still important to consider the influence diagnosis has on the assessment and treatment process, since diagnosis informs National Institute for Care Excellence (NICE) guidelines and dictates the choice of treatment in most psychiatric disorders.

Laura had long standing diagnoses of Borderline Personality Disorder (BPD), Anti-Social Personality Disorder (ASPD) and poly drug misuse. The concept of personality disorders is controversial because there has been no single definition of 'normal' or of 'abnormal' personality developed (Millon \& Grossman, 2006). Tyrer et al. (2015) said it is difficult to clearly define personality disorders, as mental illnesses because the descriptions developed are often unclear and unvalidated. Other arguments that support the idea that personality disorders do not exist include: the evidence that despite diagnoses of personality disorder, peoples' personalities are continually changing and adapting and thus are not ingrained, pervasive or persistent (Shea \& Yen, 2003); two people with the same personality disorders diagnosis will not present in the same way (Fairfax, 2011) and that personality exists on a spectrum so cannot be classified as 'normal' or 'abnormal' (Tyrer et al., 2015).
Despite these controversies, defining personality disorders and using these diagnostic descriptions can be useful for clinicians as it provides a shared language (Claridge \& Davis, 2003) which can be used to collaboratively discuss a particular client or group of clients' difficulties and develop meaningful interventions. In addition, having categories for different personality disorders that list and describe common symptoms can be a useful way of identifying that a person needs more assessment and support for a potential personality disorder diagnosis. Finally, receiving a definitive diagnosis can be a relief for service users as it may allow them to further understand and identify their difficulties and seek support from peers and specialised services, many of which require a diagnosis as part of their criteria (Department of Health, 2014).

There have been many attempts to explain the problems that people such as Laura exhibit to understand how personality difficulties emerge. Laura, like many people with diagnosed personality disorder(s) (particularly BPD) had a traumatic childhood that included emotional /sexual abuse and neglect; it has been suggested that the symptoms seen are a result of coping with this trauma (Laporte et al., 2011). This explanation particularly fits Laura's case because she experienced early trauma in her childhood, which was then compounded by trauma later in life (Newrith et al., 2006). However, the causal relationship between childhood trauma and the development of personality disorder traits is not necessarily strong and Berenz et al. (2013) argues that more research needs to be conducted in this area, especially since their study had a number of limitations including small sample size. 
Disturbed attachment in childhood, which fundamentally alters a person's personality development, is another common theory (Bowlby, 1969). It is thought that insecure attachment in childhood may affect interpersonal relationships in adulthood (Department of Health, 2015). Laura described that her mother did not love her and also had chaotic relationships with other family members including her father and siblings. Both ASPD and BPD have been described in terms of attachment theory; it is thought that maladaptive schemas and styles of attachment developed in childhood relating to attachment could explain the traits seen in many personality disorders (Levy et al., 2015). However, these theories also threaten the validity of personality disorder diagnosis as, according to Levy et al. (2015) BPD can be explained by an anxious attachment style whilst ASPD arises from an avoidant attachment style both of which develop in different ways. Furthermore, Laura presented as violent at times and this may be explained by her observing and imitating violence she saw in her childhood; she has thus learnt to incorporate this into how she lives her life (Bandura, 1969; Newrith et al., 2006). Turner et al. (2011) argue that if Laura can begin to understand where her personality difficulties may have emerged from, she can begin to work towards a meaningful recovery.

\section{Formulation}

The DSM-V (APA, 2013) recommends using clinical case formulation when assessing service users for personality disorders because clinical judgement is required to understand if symptoms represent a pathological condition that requires treatment/support. Formulation is the development of a narrative that combines the different factors and aspects of a person's life that contributes to the mental health problems they present with. It also focuses on the positive aspects of that person, their life and their skills, which could be utilised in the care planning and recovery process (MacNeil et al. 2012). Laura's historical diagnoses of BPD and ASPD were confirmed when she was transferred from prison to the secure unit after a lengthy referral and assessment process by the prison mental health team and the MDT at the secure unit.

A 5P'S formulation (MacNeil et al., 2012; Kuyken et al., 2014) was developed (see table one). Formulation is a psychologically informed approach that is vital within mental health care and is widely used throughout services (British Psychological Society, 2011). The formulation developed shows that Laura has significant difficulties in controlling her emotions, developing and sustaining relationships with others, feeling rejected, acting on impulsive thoughts, forensic history and managing aggression/violence towards others. These are all traits that indicate the present of BPD (Skodol et al., 2011; Biskin \& Paris, 2012; Few et al., 2016) and ASPD (Verona \& Patrick, 2015; Few et al., 2015).

\section{Interventions}

The unit that Laura was treated on had its own evidence-based model of care based on the idea that women with complex mental health problems need personalised treatment that considers all of their needs holistically in order to support them through recovery, allowing them to feel empowered. There is also emphasis placed on risk assessment, particularly for those service users with a forensic history (National Institute for Mental Health in England, 2003). For Laura, this meant that the staff on 
Presenting Problems - describe the main problems that person is currently requiring support for

- Long term diagnoses of borderline personality disorder, antisocial personality disorder and poly drug misuse.

- Laura often attempted to manage symptoms of her distress by requesting medications designed to alleviate anxiety.

- Severe difficulties in managing emotions, especially anger and frustration.

- Difficulties in maintaining relationships and trusting others. Often Laura will use the relationships she has with people as a means to get her needs met.

- Frequent use of ligatures to cope with emotions and thoughts.

- Varied relationships with staff: some appropriate, some over familiar and some volatile and hostile.

- Laura can become verbally threatening and sometimes physically aggressive/violent towards staff and peers at times. On occasions this has led to her needing to be placed in seclusion or on focused observation levels.

\section{Precipitating Factors - events or circumstances that may have triggered the} development of current problems.

- Laura has long standing diagnoses of borderline personality disorder and antisocial personality disorder

- She has sporadic contact with her family.

- Laura doesn't feel she needs to be in hospital

- She often blames the nursing staff for "making (her) ill"

- Laura describes having sudden, violent intrusive thoughts that are unwanted and distressing

- Laura often requests unrealistic things from nursing staff, if these requests are declined it can lead to hostility.

- Laura misses her family

- She has variable relationships with peers on the unit.

Predisposing Factors -factors that have made the person vulnerable to developing mental health problems and associated symptoms. These may be biological or childhood factors.

- Had difficulties making friends at school and was bullied. Laura would react by being physically violent and consequently was excluded.

- Aggressive, alcoholic father

- Describes feeling like her mother didn't love her

- Sexually abused from a young age

- Using a variety of illegal drugs from a young age

- Has been in many abusive relationships since her late teens.

- Comes from a socially and economically deprived background

- Long forensic history 
Perpetuating Factors - these are factors that maintain the current problems.

- Laura does not want to engage in formal therapy and really struggles to build and maintain therapeutic relationships with the nursing staff and members of the MDT

- She continues to feel that she needs sedating medications

- Over the years Laura's engagement with mental health services has been very sporadic. She will often engage with teams briefly before cutting contact and pulling away.

- Members of staff are guarded in their interactions with Laura because they feel threatened by her.

- Laura often has unrealistic demands and feels let down by the service when these are not met.

- The MDT currently will not let Laura attend occupational therapy sessions because of concerns over her previous aggressive behaviour towards staff and others.

- Laura is often worried about court dates, charges against her, tribunal dates etc

Protective Factors - these are factors that moderate the current problems and can be utilised and enhanced during the recovery process.

- Survivor or severe trauma

- Could be classed as a 'fighter' - she has always found ways of ensuring her needs are met

- Laura wants to reconnect with her family and says she is apologetic for her past actions

- Laura is generally apologetic to staff following verbally/physically aggressive behaviour

- Laura understands and will explain that she struggles to control her frustration and anger

- Laura is keen to engage in occupational therapy sessions

- When prompted she is able to reflect on why she might have been angry/threatening/hostile.

- Recognises that she has intrusive thoughts of being violent and says that she feels like she has to remove herself from situations to protect those she cares about.

- Laura will ask for 1:1 time with nursing staff and the clinical psychologist. These are unplanned, ad hoc sessions, but she engages well during them. 
the unit attempted to understand her life experiences and current problems rather than just focusing on her diagnoses of BPD and ASPD (Jones and Wright, 2015).

This recovery-based approach was developed by considering NICE guidelines; for example, the guidelines for BPD (NICE, 2009a) suggest that any nursing team should aim to provide a consistent, boundaried approach that focuses on building strong therapeutic relationships that allow the service user to feel hope and optimism for their recovery. Furthermore, the NICE guidance for self harm (NICE, 2011), outlines that practitioners should aim to provide service users with non-judgemental care that treats each incident of selfharm separately; aiming to understand the factors that contributed to effected this episode so that a comprehensive risk management plan can be developed. In contrast, the NICE guidance for Schizophrenia (NICE, 2009b) emphasize that the development of trusting therapeutic relationships is paramount, rather than risk management. This shows the complexities of providing care for service users such as Laura and further adds to the importance of compiling a meaningful formulation to guide care interventions.

From the formulation Laura's main issues relating to her personality disorder were identified as: difficulties in managing negative emotions such as stress and anger and violence/aggression to others. Usually it is recommended that the service user is involved with the collaborative care planning process (Campbell, 2013), however the MDT carried out this process with little input from Laura. This is a criticism of the care that Laura received, but it could be argued that it was necessary to make bestinterest decisions because Laura was severely unwell (NMC, 2015). Laura was involved as much as possible and was also given the opportunity to speak to advocates and 'service user champions' to allow her to provide her views on her care.

Involving Laura in her care continued to prove difficult, as she would quickly become aggressive and accused staff of not meeting her needs. Consequently Laura was viewed as 'unmanageable' and some of the more inexperienced staff members started to feel like Laura was 'putting it on'. Negative attitudes towards people with a personality disorder label are well documented (Newton-Howes et al., 2008; Clarke, 2015) and impact on the quality of care that service users receive (Department of Health, 2014).

Temporary staff and inexperienced staff on the unit sometimes had less of an understanding of Laura's needs compared to other service users whom they saw as less 'troublesome'. Educating staff on the nature of personality disorders so that they are able to understand where Laura's difficulties arise from may help change these attitudes (Department of Health, 2009). Fanaian et al. (2013) interviewed 60 clinicians who worked and had an interest in personality disorders; these clinicians identified that education for staff working in this area was vital to improve care for service users. Additionally, Krawitz (2004) found that from 418 participants training was effective in achieving positive attitudinal change towards people with borderline personality disorder. This training would also help strong therapeutic relationships to develop which have been shown to enhance recovery (Gillard et al., 2015). On the other 
hand, the experienced nurses on this unit were generally good at discouraging negativity by fostering compassionate, recovery-focused values. During handovers, a Safewards approach (Bowers, 2014) was used and nursing staff endeavoured to say something positive about each service user or explain negative behaviours psychologically.

This was a very challenging time for the unit and there were issues with relational security due to the dynamics on the ward and service user mix. This may have exacerbated negative countertransference, which is an alternative explanation for negative attitudes amongst staff. Negative countertransference occurs when staff members take on the emotional distress of a service user in an unconscious manner (King, 2014). This causes them to retreat from the service user and thus potentially damage the therapeutic relationship (Liebman \& Burnette, 2013), leading to poorer outcomes. One way of managing and preventing counter transference is to utilise clinical supervision sessions (Bland \& Rossen, 2005). Clinical supervision can help to: reduce stress, develop knowledge and encourage reflective practice (Cookson et al., 2014).

All of the qualified nurses on the unit could access clinical supervision with another experienced nurse as advised by the Department of Health (2006). Although these sessions were often cancelled because the unit was short staffed and sessions were not deemed as high of a priority compared to other ward tasks; this phenomenon has been found to be common in health services (Long et al., 2014). Since there was a high rate of sickness within this unit, ensuring that nurses were able to attend supervision was vital to prevent burnout (Department of Health, 2015).
Furthermore, ad hoc group supervision sessions after incidences or when stress levels were high were often provided. These sessions were conducted by a facilitator and were mainly beneficial; however, sometimes it was found that staff members used them to vent their feelings. Daykin and Gordon (2010) believe that team based supervision sessions should allow participants to talk through their emotions in a nonjudgemental manner, whilst containing them and guiding the development of solutions rather than enhancing a blame culture. Buus et al. (2012) recommend implementing a session agenda for clinical supervision sessions, agreed by all in attendance, to ensure everyone gets chance to contribute so that meaningful solutions are found.

Livesley (2005) described five phases for helping people with personality disorders; Laura's current treatment fits into the second step (containment) for which the aims are to stabilize emotions, manage distress and provide structure whilst starting to build therapeutic relationships. Additionally, Shepherd et al. (2016) explain that providing safety and security is important for future recovery. Ultimately it was hoped that Laura could begin to develop an understanding of her emotions and resulting behaviours and engage in low-stimulus activities to occupy her time and reduce boredom (Green \& Robinson, 2005), which Laura has said can trigger agitation. A violence reduction care plan was developed to aid Laura and the team to manage her distress by recognising her early warning signs and developing resources for de-escalation and providing Laura with reassurance. Particular emphasis was placed on team members knowing how to respond to persistent, unrealistic 
requests from Laura, as providing a consistent team approach has been shown to help service users who have a diagnosis of personality disorder (Beckley, 2010). Therefore the care plan gave example responses for common requests that Laura would have, allowing staff to feel confident in their approach. Excerpts from this care plan are shown in table two.

This care plan was difficult to implement successfully as there were a large number of temporary staff that would not return for long periods of time and so there was a lack of consistency in the delivery of the interventions which is a common issue seen across health services (Hurst \& Smith, 2011). Thus on several occasions Laura became violent/aggressive in situations where consistent, quality care may have prevented it (Hurst \& Smith, 2011). The unit therefore needs to provide a more consistent staff team and prevent burnout in order to ensure that service user's care plans are being effectively implemented and followed by all. However, this may be challenging to implement in practice due to funding constraints and budget pressures that occur irrespective of the changing levels of acuity on mental health units. A consistent staff team would also aid in the development of therapeutic relationships (Sainsbury, 2010), which Bowlby (1988) said could provide the stable, secure attachment that Laura has likely been lacking until she was admitted to the unit. Additionally, care plans for individual service users should be better communicated to staff members who may be unfamiliar with them to improve patient care and safety (Kanerva et al., 2013).

Low-stimulus activities were also provided to prevent Laura from becoming bored and thus frustrated
(Green \& Robinson, 2005). Unfortunately, due to the risk and likelihood of violence, Laura could not attend group occupational therapy sessions; instead, Laura was able to have 1:1 sessions such as crafts on the unit. This flexible approach allowed Laura to feel valued and she found these activities beneficial. Furthermore, Laura found exercise to be good at relieving stress so she was given access to sports facilities (Wynaden et al., 2012). Regrettably these sessions sometimes had to be rescheduled because staffing levels reduced or due to an increase in ward acuity at short notice. This meant that Laura felt there lack of reliability which compounded her sometimes disparaging views of the nursing team and damaged therapeutic relationships (Dziopa and Ahern, 2008). To combat this, activity planners could be used that would allow the team to know how many staff members were needed in advance so as not to disappoint Laura (Hutcheson et al., 2010).

\section{Outcomes}

During the first six months of her treatment on the unit the number of aggressive/self-harm incidents Laura was involved in decreased and she appeared more able to verbalise her frustrations and inform staff when she was feeling agitated/upset. This confirms that boundaried therapeutic relationships were developing positively between Laura and the care team, whom were therefore able to understand the reasons why Laura became frustrated (Department of Health, 2015). Furthermore, Laura was more able to reflect on specific incidences and pre-emptively discuss her views on situations before they became problematic (NICE, 2009a). Overall, Laura is in the early stages of 
Table 2: Excerpts from violence reduction care plan

\section{Who/what is the typical target of this aggression?}

- Laura is often verbally aggressive towards staff if she feels her needs are not being met.

- She can also become paranoid and believe that staff are not being truthful to her or are being inconsistent in their treatment of her.

\section{Early Warning Signs}

- Having an audience of staff listen to her complaints (limit this to 1 wherever possible)

- Laura stating following phrases: "I am having thoughts of hitting someone" or "I feel like doing something"

- Clenching fists, hitting walls/doors

- If Laura has used all of her PRN medication for the day/night this will often cause her to become agitated later on when she wants more medication

\section{Preventing Aggression}

- Limit number of staff in communal areas, as the more staff around, Laura seems to increase the volume and aggressiveness of her behaviour

- Staff not to get in to discussions about trigger issues, instead listen and allow Laura to vent

- Staff to try and explain to Laura how much PRN medication she has left (if any) to attempt to prevent later agitation surrounding this

- Staff should try and distract Laura - get her to watch TV, listen to music etc

\section{Example responses}

The following are examples of responses that can be given to Laura to help prevent aggression when she is making certain requests. The aim is to provide her with consistent team responses:

\section{1.) Medication}

1. Asks for more PRN

2. Medication is not working for her and needs reviewing by the Doctor, or requests to see the Doctor

Response: Medication is reviewed every week by the consultant all queries to be forwarded to consultant

\section{2.) Social Work}

Wants to see social worker

Response: Social worker is aware of the requests and will provide feedback every week after ward round 
her treatment and the course of her stay at the unit will largely depend on the result of her court case and the subsequent section (if any) of the MHA (1983, as amended 2007) she is placed on. However, if she were to stay on the unit a more in depth, recoveryfocused care pathway would be developed (Doyle et al., 2012) with Laura that outlines the goals of her treatment and the medical, psychological and nursing interventions that would be used.

\section{Conclusion}

In conclusion, Laura had complex needs and the staff team faced considerable challenges in assessing her and planning interventions that could be beneficial. Laura was diagnosed with BPD and ASPD and following analysis it is likely that these diagnoses did accurately describe Laura's presenting problems, although the formulation process better augmented the team's understanding of her issues. From Laura's past history it has been shown that her personality difficulties can be described in terms of attachment theory, learning theory and a post-traumatic stress reaction. It is clear that the unit attempted to follow evidenced based guidelines when planning Laura's care but that the inconsistent staff team and high level of staff burnout and sickness hindered the implementation of these plans, as is true nationally. As a result, the unit need to provide more structured supervision sessions that aim to prevent staff burnout and attempt to staff the unit with more regular workers whom Laura can build more trust with.

\section{References}

American Psychiatric Association. (2013). Diagnostic and statistical manual of mental disorders, (5th ed.).
Washington, DC: American Psychiatric Association.

Aveline, M. (1999). 'The advantages of formulation over categorical diagnosis in explorative psychotherapy and psychodynamic management', European Journal of Psychotherapy, Counselling \& Health, 2(2), pp. 199.

Bandura, A. (1969). Principles of behaviour modification. New York, NY: Holt, Rinehart and Winston.

Beckley, K. (2010). 'Making sense of interpersonal dynamics: A schema focused approach'. In: Willmot P. and Gordon N. (eds.) Working positively with personality disorder in secure settings: A practitioner's perspective, pp. 173-187. Oxford, United Kingdom: John Wiley \& Sons.

Berenz, E.C., Amstadter, A.B., Aggen, S.H., Knudsen, G.P., ReichbornKjennerud, T., Gardner, C.O., and Kendler, K.S. (2013). 'Childhood trauma and personality disorder criterion counts: A co-twin control analysis', Journal of Abnormal Psychology, 122(4), pp. 1070-1076.

Biskin, R.S. and Paris, J. (2012). 'Diagnosing borderline personality disorder', Canadian Medical Association Journal, 184(16), pp. 1789-1794.

Bland, A. R. and Rossen, E. K. (2005). 'Clinical supervision of nurses working with patients with borderline personality disorder', Issues In Mental Health Nursing, 26(5), pp. 507-517.

Bowers, L. (2014). 'Safewards: A new model of conflict and containment on psychiatric wards', Journal of Psychiatric and Mental Health Nursing, 21(6), pp. 499-508. 
Bowlby, J. (1969). Attachment and loss: Vol. 1. Attachment. New York, NY: Basic Books.

British Psychological Society. (2011). Good practice guidelines on the use of psychological formulation. Leister, United Kingdom: British Psychological Society.

Buus, N., Angel, S., Traynor, M. and Gonge, H. (2010). 'Psychiatric hospital nursing staff's experiences of participating in group-based clinical supervision: an interview study', Issues In Mental Health Nursing, 31(10), pp. 654-661.

Campbell, J., Stickley, T., Bonney, S. and Wright, N. (2013). 'Recovery as a framework for care planning'. In: Hall A., Wren M. and Kirby S. (eds.) Care Planning in Mental Health: Promoting Recovery (2nd ed.), pp. 25-51. Somerset, NJ: John Wiley \& Sons.

Clarke, S., Taylor, G., Bolderston, H., Lancaster, J. and Remington, B. (2015). 'Ameliorating patient stigma amongst staff working with personality disorder: Randomized controlled trail of self-management versus skills training', Behavioural and Cognitive Psychotherapy, 43(6), pp. 692-704.

Coid, J., Yang, M., Tyrer, P., Roberts, A., \& Ullrich, S. (2006). 'Prevalence and correlates of personality disorder in Great Britain', The British Journal of Psychiatry, 188(5), 423-431.

Cookson, J., Sloan, G., Dafters, R. and Jahoda, A. (2014). 'Provision of clinical supervision for staff working in mental health services', Mental Health Practice, 17(7), pp. 29-34.

Daykin, A., \& Gordon, N. (2010). 'Establishing a supervision culture for clinicians working with personality disordered offenders in a high secure hospital'. In: Willmot P. and Gordon N. (eds.) Working positively with personality disorder in secure settings: A practitioner's perspective, pp. 200212. Oxford, United Kingdom: John Wiley \& Sons.

Department of Health. (2006). From Values to Action: The Chief Nursing Officer's Review of Mental Health Nursing. London, United Kingdom: Department of Health.

Department of Health. (2009). Recognising complexity: Commissioning guidance for personality disorder services. London, United Kingdom: Department of Health.

Department of Health. (2014). Meeting the Challenge, Making a difference. London, United Kingdom: Department of Health.

Department of Health. (2015). Working with offenders with personality disorder. A practitioners guide. London, United Kingdom: Department of Health.

Doyle, M., Logan, C., Ludlow, A. and Holloway, J. (2012). 'Milestones to recovery: Preliminary validation of a framework to promote recovery and map progress through the medium secure inpatient pathway', Criminal Behaviour \& Mental Health, 22(1), pp. 53-64.

Dziopa, F. and Ahern, K. (2008). 'What makes a quality therapeutic relationship in psychiatric/mental health nursing: A review of the research literature.', The Internet Journal of Advanced Nursing Practice, 10(1).

Fairfax, H. (2011). 'Re-conceiving personality disorders: Adaptations on a 
dimension?', Counselling Psychology Quarterly, 24(4), pp. 313-322.

Fanaian, M., Lewis, K. L. and Grenyer, B. S. (2013). 'Improving services for people with personality disorders: Views of experienced clinicians', International Journal of Mental Health Nursing, 22(5), pp. 465-471.

Few, L. R., Lynam, D. R., Maples, J. L., MacKillop, J. and Miller, J. D. (2015). 'Comparing the utility of DSM-5 Section II and III antisocial personality disorder diagnostic approaches for capturing psychopathic traits', Personality Disorders: Theory, Research, and Treatment, 6(1), pp. 6474.

Few, L. R., Miller, J. D., Grant, J. D., Maples, J., Trull, T. J., Nelson, E. C., Oltmanns, T.F., Martin, N.G., Lynskey, M.T. and Agrawal, A. (2016). 'Trait-based assessment of borderline personality disorder using the NEO Five-Factor Inventory: Phenotypic and genetic support', Psychological Assessment, 28(1), pp. 39-50.

Gilburt, H. (2015). Mental health under pressure. London, United Kingdom: The King's Fund.

Gillard, S., Turner, K. and Neffgen, M. (2015). 'Understanding recovery in the context of lived experience of personality disorders: A collaborative, qualitative research study', $B M C$ Psychiatry, 15(183).

Green, B. and Robinson, L. (2005). 'Reducing violence in a forensic mental health unit: a seven-year study', Mental Health Practice, 9(4), pp. 4044.

Hurst, K. and Smith, A. (2011). 'Temporary nursing staff-Cost and quality issues', Journal of Advanced Nursing, 67(2), pp. 287-296.

Hutcheson, C., Ferguson, H., Nish, G. and Gill, L. (2010). 'Promoting mental wellbeing through activity in a mental health hospital', British Journal of Occupational Therapy, 73(3), pp. 121128.

Jones, E. S. and Wright, K. M. (2015). ""They're Really PD Today": An exploration of mental health nursing students' perceptions of developing a therapeutic relationship with patients with a diagnosis of antisocial personality disorder', International Journal of Offender Therapy and Comparative Criminology. 1-18.

King, G. (2014). 'Staff attitudes towards people with borderline personality disorder', Mental Health Practice, 17(5), pp. 30-34.

Krawitz, R. (2004). 'Borderline personality disorder: Attitudinal change following training.' Australian and New Zealand Journal of Psychiatry, 38, pp. 554-559.

Kuyken, W., Padesky, C.A. and Dudley, W. (2014). Collaborative case conceptualization: Working effectively with clients in cognitive-behavioral therapy. New York, NY: The Guilford Press.

Laporte, L., Paris, J., Guttman, H. and Russell, J. (2011). 'Psychopathology, childhood trauma, and personality traits in patients with borderline personality disorder and their sisters', Journal of Personality Disorders, 25, pp. 448-462.

Lean, M., Leavey, G., Killaspy, H., Green, N., Harrison, I., Cook, S. and King, M. (2015). 'Barriers to the sustainability of an intervention 
designed to improve patient engagement within NHS mental health rehabilitation units: a qualitative study nested within a randomised controlled trial', BMC Psychiatry, 15(1), pp. 110 .

Levy, K. N., Johnson, B. N., Clouthier, T. L., Scala, J. W. and Temes, C. M. (2015). 'An attachment theoretical framework for personality disorders', Canadian Psychology, 56(2), pp. 197207.

Liebman, R. E. and Burnette, M. (2013). 'It's not you, it's me: An examination of clinician- and clientlevel influences on countertransference toward borderline personality disorder', American Journal of Orthopsychiatry, 83(1), pp. 115-125.

Livesley, W. J. (2005). 'Principles and strategies for treating personality disorder', Canadian Journal of Psychiatry, 50(8), pp. 442-450.

Long, C. G., Harding, S., Payne, K. and Collins, L. (2014). 'Nursing and health-care assistant experience of supervision in a medium secure psychiatric service for women: Implications for service development', Journal of Psychiatric and Mental Health Nursing, 21(2), pp. 154-162.

MacNeil, C.A., Hasty, M.K., Conus, P. and Berk, M. (2012). 'Is diagnosis enough to guide interventions in mental health? Using case formulation in clinical practice', BMC Medicine, 10, pp. 111.

Mental Health Act. (1983). Chapter 20. London, United Kingdom: HMSO.

Mental Health Act. (2007). Chapter 12. Retrieved from http://www.legislation.gov.uk/ukpga/2 007/12/pdfs/ukpga_20070012_en.pdf
Millon, T. and Grossman, S.D. (2006). 'Millon's evolutionary model for unifying the study of normal and abnormal personality'. In: Strack S. (ed.) Differentiating Normal and Abnormal Personality, (2nd ed.), pp. 349. New York, NY: Springer Publishing Company.

National Institute of Health and Care Excellence. (2009a). Borderline personality disorder: recognition and management. London, United Kingdom: NICE.

National Institute of Health and Care Excellence. (2009b). Schizophrenia: core interventions in the treatment and Management of schizophrenia in Adults in Primary and Secondary Care. London, United Kingdom: NICE.

National Institute of Health and Care Excellence. (2011). Self-harm in over 8s: long-term management. London, United Kingdom: NICE.

National Institute for Mental Health in England. (2003). Personality Disorder: No Longer a Diagnosis of Exclusion. London, United Kingdom: NIMH(E).

Newrith, C., Meux, C. and Taylor, P.J. (2006). Personality Disorder and Serious Offending. London, United Kingdom: Hodder Education.

Newton-Howes, G., Weaver, T. and Tyrer, P. (2008). 'Attitudes of staff towards patients with personality disorder in community mental health teams', Australian and New Zealand Journal of Psychiatry, 42(7), pp. 572577.

Nursing and Midwifery Council. (2015). The Code: Professional 
standards of practice and behaviour for nurses and midwives. London, United Kingdom: The Nursing and Midwifery Council.

Royal College of Psychiatrists. (2010). 'Accreditation for Inpatient Mental Health Services.' Retrieved from https://www.google.co.uk/url?sa=t\&rct $=\mathrm{j} \& \mathrm{q}=\&$ esrc $=\mathrm{s} \&$ source $=$ web $\& \mathrm{~cd}=1 \& \mathrm{v}$ ed=0ahUKEwjRzJD5guzPAhVFDxoK HfudA8AQFggeMAA\&url=http\%3A \%2F\%2Fwww.rcpsych.ac.uk\%2Fpdf $\%$ 2FStandards\%2520for\%2520Inpatient $\% 2520$ Wards $\% 2520$ -

$\% 2520$ Working\%2520Age\%2520Adul ts\%2520-

\%2520Fourth\%2520Edition.pdf\&usg= AFQjCNG6vW6AwpRPsutjLdbCNJatOsctQ\&cad=rja

Sainsbury, L. (2010). 'Attachment theory and the therapeutic relationship in the treatment of personality disorder'. In: Willmot P. and Gordon $\mathrm{N}$. (eds.) Working positively with personality disorder in secure settings: A practitioner's perspective, pp. 93115. Oxford, United Kingdom: John Wiley \& Sons.

Shea, M.T. and Yen, S. (2003). 'Stability as a distinction between Axis I and Axis II disorders', Journal of Personality Disorders, 17, pp. 373386.

Shepherd, A., Doyle, M., Sanders, C. and Shaw, J. (2016). 'Personal recovery within forensic setting Systematic review and meta-synthesis of qualitative methods studies', Criminal Behaviour and Mental Health, 26, pp. 59-75.

Skodol, A. E., Bender, D. S., Morey, L. C., Clark, L. A., Oldham, J. M., Alarcon, R. D., Krueger, R.F., Verheul, R., Bell, C.C. and Siever, L. J. (2011). 'Personality disorder types proposed for DSM-5', Journal of Personality Disorders, 25(2), pp. 136169.

Skodol, A. E., Morey, L. C., Bender, D. S. and Oldham, J. M. (2013). 'The ironic fate of the personality disorders in DSM-5. Personality Disorders', Theory, Research, and Treatment, 4(4), pp. 342-349.

Turner, K., Lovell, K. and Brooker, A. (2011). '... and they all lived happily ever after': 'recovery' or discovery of the self in personality disorder?', Psychodynamic Practice, 17(3), pp. 341-346.

Tyrer, P., Reed, G. M. and Crawford, M. J. (2015). 'Classification, assessment, prevalence, and effect of personality disorder', The Lancet, 385(9969), pp. 717-726.

Verona, E. and Patrick, C. J. (2015). 'Psychobiological aspects of antisocial personality disorder, psychopathy, and violence', Psychiatric Times, 32(3), pp. 1-7.

World Health Organisation. (1992). 'The ICD-10 classification of mental and behavioural disorders: Clinical descriptions and diagnostic guidelines', (ICD-10) (10 ${ }^{\text {th }}$ ed.). Geneva, World Health Organisation.

Wynaden, D., Barr, L., Omari, O. and Fulton, A. (2012). 'Evaluation of service users' experiences of participating in an exercise programme at the Western Australian State Forensic Mental Health Services', International Journal of Mental Health Nursing, 21(3), pp. 229-235. 